\title{
Slide Test for Detection of Slime Formation in Vibrios
}

Some members of Enterobacteriaceae and a few other organisms like Pseudomonas aeruginosa are known to produce an extracellular, viscid, amorphous surface material known as 'slime'. Phosphates and other growth promoting substances in media can enhance production of slime. ANDERSON ${ }^{1}$ considered that the phenomenon of slime formation, which was poor or irregular on nutrient agar but abundant in presence of relatively high concentration of phosphate, was widely distributed in the family Enterobacteriaceae.

$V$. cholevae, except for a few mutants, were generally supposed not to produce any slime or capsule ${ }^{2}$. LANKFORD et $\mathrm{al}^{3}$ could, however, detect evidence of slime formation in the cultures of $\mathrm{V}$. cholerae. With a modification of Anderson's technique, Neogy and Sanyal ${ }^{4}$ succeeded in demonstrating slime in several strains of $V$. cholevae and $\mathrm{NAG}$ vibrios, although the same could not be shown convincingly in $\mathrm{El}$ Tor vibrios. Working on the analogy of ANDERSON's observations, the present communication is based on a design of a simple slide technique for detection of slime formation in different vibrio species.

Material and method. Organisms: 64 strains of vibrios were studied consisting of classical $r$. cholerae, El Tor vibrios and NAG vibrios, isolated from different sources including cases of cholera. As controls, 8 members of the family Enterobacteriaceae and 2 strains of PS. aevuginosa were also included. The strains were maintained on nutrient agar slants.

Medium: A special phosphate agar medium, very similar to that used by ANDERSON, was prepared with Lab. lemco $(0.5 \%)$, Bacto peptone, Difco $(5.0 \%)$, sodium chloride $(0.85 \%)$, Bacto agar, Difco $(3.0 \%)$ and phosphate buffer $-\mathrm{pH} 7.2$ (q.s.). After dissolving the ingredients by heating and filtering through cotton, the $\mathrm{pH}$ was checked $(7-7.2)$ and the medium was autoclaved at $15 \mathrm{lbs}$ pressure for $30 \mathrm{~min}$. Slants in tubes were prepared.

The surface of a clean, grease-free glass slide was divided into 2 halves by grease-pencil markings. A loopful of distilled water was put on to one half of the slide and sufficient growth from an overnight culture in phosphate agar medium was emulsified in the distilled water with a loop. The same procedure was repeated in a loopful of $0.5 \%$ sodium taurocholate solution on the other half of the glass slide. Overnight growth of the same organism in nutrient agar was tested in identical manner. Appearance of a slimy material (SM) almost immediately, in the form of a mucilaginous mass, was indicative of positive reaction. The SM not only moved along with the loop during the process of emulsification but also seemed to be adherent to it.

Results. All the 64 strains of vibrios, 2 strains of $P$ seudomonas and 2 strains of $E$. coli out of 8 Enterobacteriaceae produced SM in phosphate agar which could be detected by the slide test with both distilled water and $0.5 \%$ sodium taurocholate solution. There was, however, a difference in the degree of reaction between the strains, and within the same strain also; a stronger reaction was evident with $0.5 \%$ sodium taurocholate solution in most cases. Typical string formation, as observed by $\mathrm{SMITH}^{5}$ in the 'String test' for vibrio identification with a stronger lysing agent-sodium desoxycholate, was noticed in vibrio strains producing copious SM. All classical $V$. cholevae produced SM in nutrient agar detectable by slide test; nevertheless, presence of phosphate in the medium definitely enhanced production of SM by these organisms. Phosphate agar medium was, however, found to be necessary for production of SM in the case of 12 out of $34 \mathrm{El}$ Tor strains and 3 out of 13 NAG vibrios. Copious amounts of SM was produced by the Pseudomonas strains, and in one strain the surface growth in phosphate agar felt gummy during the time of removal with a loop.

Summary and conclusion. Slime production was detected by a simple slide test on a glass slide with distilled water and $0.5 \%$ sodium taurocholate solution in all the 64 strains of vibrios consisting of classical $V$. cholevae, El Tor and NAG vibrios, isolated from different sources, 2 strains of Ps. aeruginos a and 2 of the 8 strains of Enterobacteriaceae, when the organisms were grown in a special phosphate agar medium. Phosphate was essential for SM in many strains of $\mathrm{El}$ Tor and NAG vibrios. Although detectable SM was observed in nutrient agar, presence of phosphate definitely augmented its production by classical $V$. cholevae. Observation of such characteristic features in vibrios justified the consideration that the viscous material was extracellular 'slime' of the organism. This also suggests that the simple slide test as employed here can be utilized for detection of SM of intestinal bacteria after growing in medium containing suitable concentration of phosphate.

Zusammenfassung. Nachweis der Schleimbildung von Vibrionen in Enterobakterien.

K. N. Neogy and A. C. Mukherj ${ }^{6}$

Department of Bacteriology,

School of Tropical Medicine,

Calcutta 12 (India), 15 September 1969.

1 E. S. Anderson, Nature 190, 284 (1961).

2 R. Pollitzer, Cholera, World Health Organization: Monograph Series, Geneva, 43, 103 (1959).

3 C. E. Lan kford and U. Legsomburana, Proceedings of the Cholera Research Symposium (Honolulu 1965), p. 109.

4 K. N. Neogy and S. N. Sanyal, Bull. Wld. Hilth. Org. 40, 329 (1969).

5 H. Smith JR., Bact. Proc., 69 (1958), referred in O. McIntyre and J. FeeLeY, Bull. Wld. Hlth. Org. 32, 627 (1965).

6 The authors wish to convey their gratefulness to Prof. J. B. Chatterjea, School of Tropical Medicine, Calcutta, for his kind permission to publish this paper.

\section{Effect of an Azo Dye on Germination and Outgrowth of Bacillus megaterium Spores}

In previous investigations we reported on the bacteriostatic effect of some stains ${ }^{1}$. In the present study we wish to investigate the action of an azo dye, gentian violet, on the development of a bacterial spore into a vegetative cell, which is known to occur in 3 phases. The first, not necessarily with all spores, is activation, by which the dor- mant spore becomes able to germinate. The second is a catabolic process, by which spore materials are degraded.

\footnotetext{
1 A. Nacci, A. E. Brusca, A. Rocca, G. Natalizi and A. Colloca, Atti Soc. med.-chir. Messina. 3, 1 (1968).
} 